\title{
Addressing the Challenge of Atrial Fibrillation Management: How to Differentiate the Approach Depending on Left Ventricular Ejection Fraction
}

\author{
Renato De Vecchis ${ }^{\mathrm{a}, \mathrm{d}}$, Andrea Paccone ${ }^{\mathrm{b}}$, Marco Di Maio ${ }^{\mathrm{c}}$
}

Atrial fibrillation (AF) secondary prevention encompasses a number of much-debated concepts that are not bound to strict and evidence-based rules and regulations. The societal guidelines [1] essentially grant the treating physician a wide discretionality concerning the type of strategy to be adopted in patients with a history of one or more episodes of AF converted to sinus rhythm. Importantly, for the management of AF patients, the classical conflict between two operational approaches still applies: rhythm control strategy versus the rate control strategy.

The first approach is aimed at the preservation of sinus rhythm, achievable with the use of antiarrhythmics, more precisely class Ia, Ic and III drugs of Vaughan Williams classification, or with ablation of atrial arrhythmogenic foci carried out with radiofrequency or cryo balloon.

On the contrary, rate control strategy is equivalent to leaving the patient in AF paying attention to keep the ventricular response as regular as possible and trying to restrain it between 60 and 90 beats/min at rest. To achieve this goal, the use of dromotropic depressant drugs at the level of the atrioventricular node (in particular verapamil or beta blockers or digoxin) is sufficient, combined with scrupulous anticoagulation.

Since several studies during the 2000s [2] established that all-cause death as well as hospitalizations did not show significant differences in the comparison between the rhythm control strategy and the rate control strategy, many scholars have become confident in the fact that rate control can play a role of preferred approach [3] due to the fact that the latter does not expose the patients to the risk of proarrhythmic events.

In truth, there has been a resizing of the concept that the

Manuscript submitted June 8, 2019, accepted July 9, 2019

aPreventive Cardiology and Rehabilitation Unit, DSB 29 "S. Gennaro dei Poveri Hospital", via S.Gennaro dei Poveri 25, 80136 Naples, Italy

bDepartment of Cardiology, University of Bari “Aldo Moro", Bari, Italy

'Department of Cardiology, University of Campania "Luigi Vanvitelli”, 80138

Naples, Italy

${ }^{\mathrm{d} C o r r e s p o n d i n g ~ A u t h o r: ~ R e n a t o ~ D e ~ V e c c h i s, ~ P r e v e n t i v e ~ C a r d i o l o g y ~ a n d ~ R e h a-~}$ bilitation Unit, DSB 29 "S. Gennaro dei Poveri Hospital", via S.Gennaro dei Poveri 25, 80136 Naples, Italy. Email: devecchis.erre@virgilio.it

doi: https://doi.org/10.14740/cr896 restoration of sinus rhythm would bring a fundamental benefit to the pump function through the retrieval of the atrial mechanical contribution to the end-diastolic ventricular filling. This concept should in fact be limited to the heart of patients free from systolic heart failure, in which a preload augmentation increases the stroke-volume, in accordance with the FrankStarling law.

However, a number of patients with recurrent AF episodes suffer from heart failure with reduced left ventricular ejection fraction (LVEF), so-called heart failure (HF) with reduced ejection fraction (HFrEF) patients, whose LVEF is less than $40 \%$ by definition. In this subset, the supplemental preload received by means of the atrial contraction at the end of ventricular diastole does not increase the force of contraction of the myocardial fibers during systole.

Indeed, in these patients, whose heart works on the flat branch of the Frank-Starling curve, the efficiency of the cardiac pump which is in essence the stroke-volume pushed into the aorta at each beat, is not increased by a restoration of the contribution of atrial systole to ventricular filling. Moreover, according to Laplace's law, augmentation of ventricular cavity dimensions increases the wall tension as well as the work for every single heart beat. Probably this might be the explanation why the loss of the atrial contractile function would seem to have little or no influence on the clinical condition and prognosis of HFREF patients [4].

An increase in the risk of death and hospitalizations would be vice versa detectable in the case of AF occurring in midrange (HFmrEF 40-49\%) and preserved ( $\mathrm{HFpEF} \geq 50 \%$ ) LVEF[3]. Therefore an appropriate operational algorithm should provide for a rate control regimen for HFrEF patients, while in HFmrEF and HFpEF patients the conversion to and maintenance of sinus rhythm should be pursued and defended using the rhythm control strategy, and also with the AF ablation when a first pharmacological approach has been tested without success.

These considerations imply the need for a profound rethinking of the current guidelines for the management of AF [1], which should be updated accordingly.

\section{Acknowledgments}

None to declare. 


\section{Financial Disclosure}

All authors declare that the present article has not benefitted from any source of funding.

\section{Conflict of Interest}

All authors do not have any conflict of interest to declare concerning the present article.

\section{Informed Consent}

Not applicable.

\section{Author Contributions}

RDV and AP contributed to conceptualization; RDV contributed to data curation; RDV, AP and MDM contributed to writing and original draft preparation; RDV, AP and MDM contributed to writing, review and editing.

\section{References}

1. Kirchhof P, Benussi S, Kotecha D, Ahlsson A, Atar D, Casadei B, Castella M, et al. 2016 ESC Guidelines for the management of atrial fibrillation developed in collaboration with EACTS. Eur Heart J. 2016;37(38):28932962.

2. Roy D, Talajic M, Nattel S, Wyse DG, Dorian P, Lee KL, Bourassa MG, et al. Rhythm control versus rate control for atrial fibrillation and heart failure. N Engl J Med. 2008;358(25):2667-2677.

3. De Vecchis R, Di Maio M, Soreca S, Ariano C. Rate control yields better clinical outcomes over a median follow-up of 20 months compared to rhythm control strategy in patients with a history of atrial fibrillation: a retrospective cohort study. Cardiol Res. 2019;10(2):98105.

4. Zafrir B, Lund LH, Laroche C, Ruschitzka F, CrespoLeiro MG, Coats AJS, Anker SD, et al. Prognostic implications of atrial fibrillation in heart failure with reduced, mid-range, and preserved ejection fraction: a report from 14964 patients in the European Society of Cardiology Heart Failure Long-Term Registry. Eur Heart J. 2018;39(48):4277-4284. 